\title{
Simultaneous Application of Dissolution/Precipitation and Surface Complexation/Surface Precipitation Modeling to Contaminant Leaching
}

\author{
Supporting Information \\ Manuscript \# es0486521 \\ Contains Five Tables
}
Defne S. Apul ${ }^{* 1}$, Kevin H. Gardner ${ }^{1}$, T. Taylor Eighmy ${ }^{1}$, Ann-Marie Fällman ${ }^{2}$, Rob N.J. Comans ${ }^{3}$
${ }^{1}$ Environmental Research Group, University of New Hampshire
Durham, NH 03824 USA

${ }^{2}$ The Swedish Environmental Protection Agency, S-106 48 Stockholm, Sweden

${ }^{3}$ Energy research center of the Netherlands (ECN), P.O. box1, 1755 ZG Petten, The Netherlands

"To whom correspondence should be addressed. The present address for the corresponding author is:

Defne Apul, Mail Stop 307, 2801 W. Bancroft St., Department of Civil Engineering University of Toledo, Toledo, $\mathrm{OH} 43606$ USA

Telephone: +1 4195308124

Fax: +1419 530-8116

E-mail: defne.apul@utoledo.edu 
Sample Collection and Treatment (adapted from Fällman (1997) and Fällman et al. (1999))

\section{Steel Slag Collection and Lysimeters}

The steel slag used in this study was obtained from a plant designed to produce low allow steel from scrap steel. The steel slag was generated by periodic dumping of molten material from the electric arc furnace. The slag was excavated from below the furnace and transported to an intermediate storage area while still in a hot, plastic state. It was then cooled by sprinkling. The slag was screened and magnetic materials were removed prior to sample collection.

In December 1992, approximately $10 \mathrm{~m}^{3}$ of slag was placed in specially constructed lysimeters located at the Lysimeter Facility at Swedish Geotechnical Institute (SGI). The lysimeters were constructed to dimensions of $3.0 \times 3.0 \times 1.2 \mathrm{~m}$ so that the largest particle size was less than $1 / 10$ the width of the lysimeter. The walls of the lysimeter were made with plywood and covered with a preformed HDPE liner. A geotextile with a fixed synthetic drainage layer was used to prevent fines from infiltrating the leachate collection lines. The leachate was collected at the center bottom of the lysimeter and directed through a PVC pipe to the basement of the adjacent SGI building. A water trap was used to prevent the atmosphere from entering the pipe. A tipping bucket system was used to measure leachate quantity over time. Leachate was then collected from a proportional sampler maintained under Ar to minimize the impact of $\mathrm{O}_{2}$ or $\mathrm{CO}_{2}$ on leachate quality. 
In October 1997, a trowel was used to carefully excavate samples. The footprint of the excavation was $0.7 \times 0.7 \mathrm{~m}$ at the lysimeter surface. The samples used in the modeling calculations were collected within the top $0.15 \mathrm{~m}$ of the surface of the lysimeter.

Samples were sieved for $4 \mathrm{~mm}$, placed in a bucket under $\mathrm{N}_{2} / \mathrm{Ar}$ and mixed prior to storage in $2 \mathrm{~L}$ plastic bags under $\mathrm{N}_{2} / \mathrm{Ar}$. Then, they were transported to a $\mathrm{N}_{2}$ glovebox for drying in the presence of Drierite.

pH-dependent Leaching

Constant $\mathrm{pH}$ experiments were done at $\mathrm{pH} 6,8,10$, and 12 , at a liquid to solid ratio of 5 $\mathrm{L} / \mathrm{kg}$ for 24 hours at room temperature. $50 \mathrm{~g}$ of dried $<4 \mathrm{~mm}$ material was placed in a $1000 \mathrm{ml}$ polyethylene beaker with about $230 \mathrm{ml}$ of deionised water. $\mathrm{pH}$ was kept constant ( $\pm 0.05 \mathrm{pH}$ units) using the Radiometer TIM 90 titrator by constantly measuring and adding $\mathrm{HNO}_{3}(0.1$ to $0.5 \mathrm{M})$ or $\mathrm{NaOH}(0.5 \mathrm{M})$ depending on the need. A magnetic propeller was used to ensure mixing. All beakers were acid cleaned prior to their use by soaking in $6 \mathrm{M} \mathrm{HCL}$ for two weeks and then $0.05 \mathrm{M} \mathrm{HNO}_{3}$ for one week.

\section{Availability Test}

Both the availability and the oxidized availability tests were performed on raw steel slag samples. The availability test used was based on the Dutch availability test, NEN 7341 . Eight grams of the crushed and milled material $(95 \%<125 \mathrm{um})$ was leached at a liquid to solid ratio of $100 \mathrm{ml} / \mathrm{g}$, first at $\mathrm{pH} 7$ for three hours, and then at $\mathrm{pH} 4$ for four hours. Filtering was carried out between the two steps, and the filter residues were added in the second step. A pH-stat titrator was used for $\mathrm{pH}$ control by adding $0.5 \mathrm{M} \mathrm{HNO}_{3}$. 


\section{Oxidized Availability Test}

Oxidized availability was measured by performing the availability test under oxidizing conditions. The same redox potential as obtained in ultra pure water in contact with the atmosphere at the same $\mathrm{pH}$ was achieved by the addition of $\mathrm{H}_{2} \mathrm{O}_{2}(30 \%)$ prior to the addition of acid. The oxidizing conditions were maintained and controlled during leaching by further addition of $\mathrm{H}_{2} \mathrm{O}_{2}$ until no change in redox potential was observed.

\section{Surface Complexation Model (SCM)}

The generalized two-layer model describes sorption as the formation of coordinative bonds between cationic/anionic sorbates and low and high affinity surface functional groups of hydrous oxides.

\section{Cation surface complexation:}

$\equiv \mathrm{FeOH}^{0}+\mathrm{Me}^{2+} \leftrightarrow \equiv \mathrm{FeOMe}^{+}+\mathrm{H}^{+}, \mathrm{K}_{\mathrm{Me}}{ }^{\text {app }}$

\section{$\underline{\text { Anion surface complexation: }}$}

$\equiv \mathrm{FeOH}^{0}+\mathrm{A}^{3-}+\mathrm{H}^{+} \leftrightarrow \equiv \mathrm{FeA}^{2-}+\mathrm{H}_{2} \mathrm{O}, \mathrm{K}_{\mathrm{A}}^{\text {app }}$

While only the general form of the equations is shown, separate equations and sorption constants were used for low and high affinity sites. To convert conditional or "apparent" complexation constants to intrinsic constants and therefore account for the variable 
charge on the oxide surface, the Coulombic correction factor calculated from the GuoyChapman theory was used:

$$
\mathrm{K}_{\mathrm{Me}}{ }^{\mathrm{app}}=\mathrm{K}_{\mathrm{Me}}{ }^{\text {int }}(\exp (-\Delta \mathrm{zF} \psi / \mathrm{RT})
$$

where, $\Delta \mathrm{z}$ is the net change in the charge number of the surface species and $\psi$ is the surface potential).

\section{Surface Precipitation Model (SPM)}

SPM provides a thermodynamic construct for a continuum between surface complexation and bulk solid precipitation (Farley et al., 1985) and Zhu (2002). What is meant by a continuum is the formation of a solid solution whose composition varies continuously between that of the original solid and a pure precipitate of the sorbing cation. To our knowledge, there has not been any other models hypothesized for describing this continuum, but it is clearly a 'real' phenomenon that has been observed spectroscopically by Sposito (1986), Zachara et al. (1989), Lower et al. (1998), Fuller et al. (2002), and Stipp et al. (1992).

Surface precipitation is an extension of the surface complexation model for high concentrations of sorbates and SPM reduces to SCM at low concentrations of sorbates. In surface precipitation, hydrous ferric oxide and metals are co-precipitate on the surface as a solid solution. Effects of surface precipitation were modeled by adjusting (in the 
feo-dlmSP database in Visual Minteq) the surface complexation constants by the solubility product for ferric hydroxide:

$\equiv \mathrm{FeOH}^{0}+\mathrm{Me}^{2+}+2 \mathrm{H}_{2} \mathrm{O} \leftrightarrow \mathrm{Fe}(\mathrm{OH})_{3(\mathrm{~s})}+\equiv \mathrm{MeOH}_{2}{ }^{+}+\mathrm{H}^{+},{ }^{*} \mathrm{~K}_{\mathrm{Me}}{ }^{\mathrm{app}}=\mathrm{K}_{\mathrm{Me}}{ }^{\mathrm{app}} \times \mathrm{K}_{\mathrm{spFe}}$

Reactions for the surface precipitation of the cation (e.g. $\mathrm{Pb}(\mathrm{OH})_{2(\mathrm{ss})}, \mathrm{Zn}(\mathrm{OH})_{2 \text { (ss), }}$, and $\left.\mathrm{Fe}(\mathrm{OH})_{3(\mathrm{~s})}\right)$ on $\mathrm{HFO}$ include a fictitious component $\mathrm{T}_{\mathrm{s}}$ required for solid-phase activity corrections:

$\equiv \mathrm{FeOH}^{0}+\mathrm{Fe}^{3+}+3 \mathrm{H}_{2} \mathrm{O}+\mathrm{T}_{\mathrm{s}} \leftrightarrow \mathrm{Fe}(\mathrm{OH})_{3(\mathrm{~s})}+\equiv \mathrm{FeOH}^{0}+3 \mathrm{H}^{+}, 1 / \mathrm{K}_{\mathrm{spFe}}$

$\equiv \mathrm{MeOH}_{2}{ }^{+}+\mathrm{Me}^{2+}+2 \mathrm{H}_{2} \mathrm{O}+\mathrm{T}_{\mathrm{s}} \leftrightarrow \mathrm{Me}(\mathrm{OH})_{2(\mathrm{~s})}+\equiv \mathrm{MeOH}_{2}{ }^{+}+2 \mathrm{H}^{+}, 1 / \mathrm{K}_{\mathrm{spMe}}$

These reactions suggest that lower $\mathrm{H}^{+}$concentrations (higher $\mathrm{pH}$ ) cause more surface precipitates to form. Hence, it is expected that surface precipitation will become more important at higher $\mathrm{pH}$ values. The $\mathrm{T}_{\mathrm{s}}$ values representative of the total surface precipitate concentrations also support this concept. The $\mathrm{T}_{\mathrm{s}}$ values for $\mathrm{pH}$ values of 6,8 , 10 , and 12 were 5.0E-05, 1.5E-04,1.7E-04, and 2.0E-04 respectively. Higher $\mathrm{T}_{\mathrm{s}}$ values at higher $\mathrm{pH}$ mean that more surface precipitates were there at high $\mathrm{pH}$ values.

\section{Tables Providing More Information on Model Runs}

Table 1 Summary of All Geochemical Simulations 


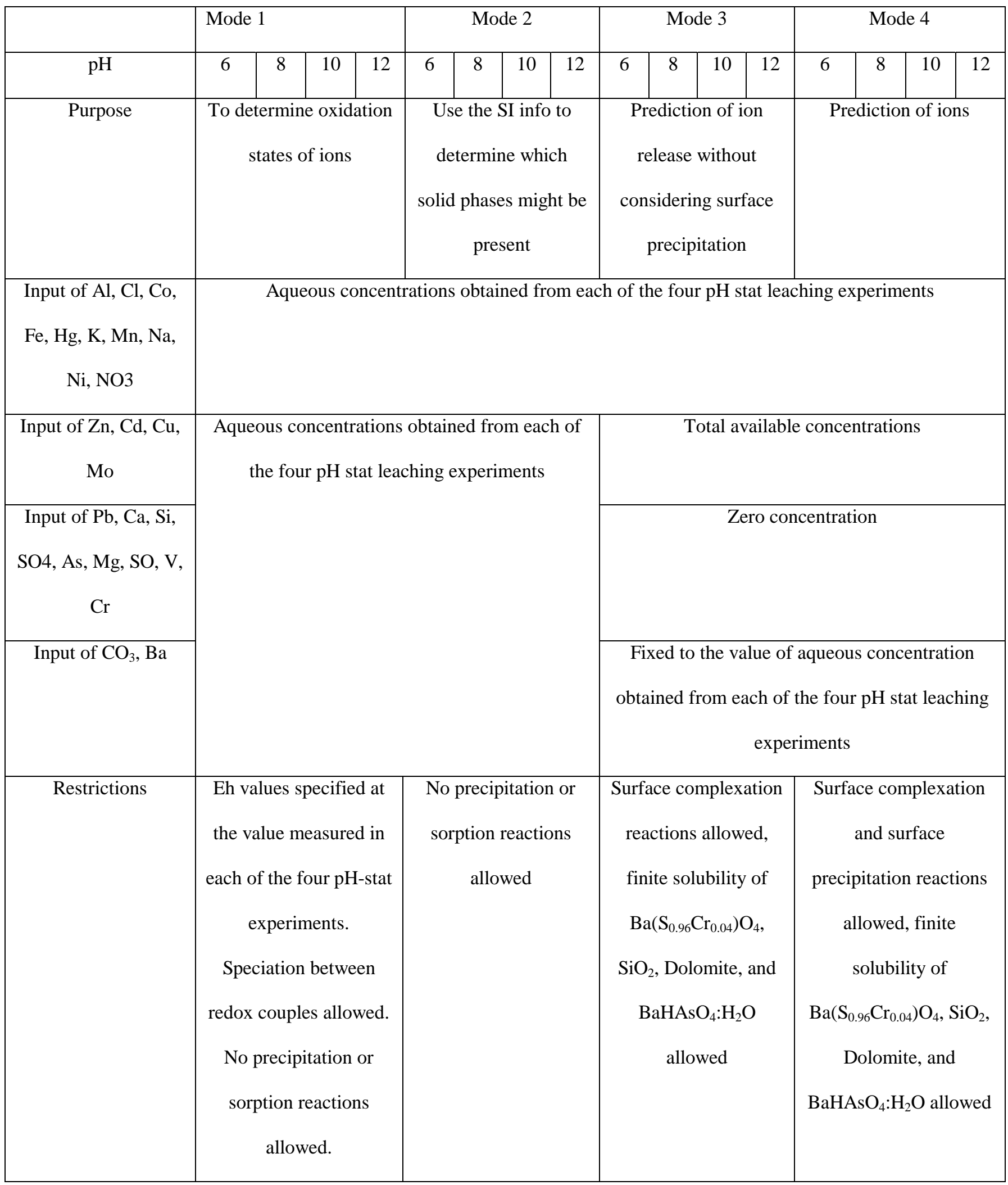


Table 2. Input Parameters for Mode 4. Background analytes are shown in Bold.

\begin{tabular}{|c|c|c|c|c|}
\hline \multirow[t]{2}{*}{$\mathrm{pH}$} & 6 & 8 & 10 & 12 \\
\hline & \multicolumn{4}{|c|}{ Concentration (M) } \\
\hline $\mathbf{A l + 3}$ & 8.15E-06 & 1.98E-08 & 4.41E-08 & 1.36E-04 \\
\hline $\mathrm{AsO} 4-3$ & \multicolumn{4}{|c|}{0} \\
\hline $\mathrm{Ba}+2$ & $4.83 \mathrm{E}-05$ & $8.15 \mathrm{E}-06$ & $1.56 \mathrm{E}-06$ & $1.30 \mathrm{E}-07$ \\
\hline $\mathrm{Ca}+2$ & \multicolumn{4}{|c|}{0} \\
\hline Cl-1 & 7.05E-03 & 9.03E-03 & 1.02E-02 & $5.08 E-03$ \\
\hline $\mathrm{Co}+3$ & 3.05E-07 & 2.22E-09 & 8.48E-10 & 8.48E-10 \\
\hline $\mathrm{Fe}+3$ & $1.12 \mathrm{E}-05$ & $1.09 \mathrm{E}-07$ & $1.02 \mathrm{E}-07$ & $5.01 \mathrm{E}-07$ \\
\hline $\mathrm{Hg}(\mathrm{OH}) 2$ & $1.20 \mathrm{E}-10$ & $2.30 \mathrm{E}-10$ & $4.99 \mathrm{E}-11$ & $1.25 \mathrm{E}-09$ \\
\hline$K+1$ & 6.06E-03 & 7.57E-03 & 8.08E-03 & 2.55E-03 \\
\hline $\mathrm{Mg}+2$ & \multicolumn{4}{|c|}{0} \\
\hline $\mathrm{Mn}+2$ & $3.66 \mathrm{E}-04$ & $5.70 \mathrm{E}-06$ & $2.06 \mathrm{E}-08$ & 1.01E-07 \\
\hline $\mathrm{Na}+1$ & 2.01E-04 & 2.04E-04 & $2.46 \mathrm{E}-03$ & 3.16E-02 \\
\hline $\mathrm{Ni}+2$ & 3.92E-06 & 2.13E-08 & 2.13E-08 & 2.13E-08 \\
\hline $\mathrm{H} 4 \mathrm{SiO} 4$ & \multicolumn{4}{|c|}{0} \\
\hline $\mathrm{SO} 4-2$ & \multicolumn{4}{|c|}{0} \\
\hline $\mathrm{VO} 2+1$ & \multicolumn{4}{|c|}{0} \\
\hline NO3-1 & 1.65E-01 & 2.00E-06 & $5.00 \mathrm{E}-05$ & 4.77E-04 \\
\hline $\mathrm{CO} 3-2$ & 8.33E-07 & $1.42 \mathrm{E}-02$ & $1.00 \mathrm{E}-05$ & $1.00 \mathrm{E}-05$ \\
\hline $\mathrm{Cd}+2$ & & $2.28 \mathrm{H}$ & & \\
\hline $\mathrm{Cu}+2$ & \multicolumn{4}{|c|}{ 3.12E-06 } \\
\hline $\mathrm{CrO} 4-2$ & $0.00 \mathrm{E}+00$ & $0.00 \mathrm{E}+00$ & $0.00 \mathrm{E}+00$ & $0.00 \mathrm{E}+00$ \\
\hline MoO4-2 & \multicolumn{4}{|c|}{$9.80 \mathrm{E}-06$} \\
\hline $\mathrm{Pb}+2$ & \multirow{2}{*}{\multicolumn{4}{|c|}{$\begin{array}{c}0 \\
150 \mathrm{E}-04\end{array}$}} \\
\hline $\mathrm{Zn}+2$ & \multicolumn{3}{|c|}{$1.50 \mathrm{E}-04$} & \\
\hline $\begin{array}{c}\text { Low Affinity Surface } \\
\text { Site }\end{array}$ & \multicolumn{4}{|c|}{$1.77 \mathrm{E}-03$} \\
\hline $\begin{array}{l}\text { High Affinity } \\
\text { Surface Site }\end{array}$ & \multicolumn{4}{|c|}{ 4.42E-05 } \\
\hline PSIo(1) & 0 & 0 & 0 & 0 \\
\hline Ts1-Fer & $5.00 \mathrm{E}-05$ & $1.50 \mathrm{E}-04$ & $1.70 \mathrm{E}-04$ & $2.00 \mathrm{E}-04$ \\
\hline $\mathrm{Pb} 3(\mathrm{VO} 4) 2$ & \multicolumn{4}{|c|}{$6.23 \mathrm{E}-05$} \\
\hline BaHAsO4:H2O & \multicolumn{4}{|c|}{$1.43 \mathrm{E}-04$} \\
\hline $\mathrm{SiO} 2$ (am,gel) & \multicolumn{4}{|c|}{$4.00 \mathrm{E}-02$} \\
\hline $\begin{array}{l}\text { Dolomite } \\
\text { (disordered) }\end{array}$ & \multicolumn{4}{|c|}{$3.72 \mathrm{E}-02$} \\
\hline $\mathrm{Ba}(\mathrm{S} 0.96 \mathrm{Cr} 0.04) 04$ & \multicolumn{4}{|c|}{$1.43 \mathrm{E}-04$} \\
\hline
\end{tabular}


Table 3. Pure phase and solid solutions added to the Visual MINTEQ database.

\begin{tabular}{|l|l|l|l|}
\hline & Log $\mathrm{K}$ & Source & $\begin{array}{l}\text { Specific Database Added } \\
\text { To }\end{array}$ \\
\hline $\mathrm{Fe}_{2}\left(\mathrm{MoO}_{4}\right)_{3(\mathrm{~s})}$ & 35.3 & Meima and Comans (1998) & Type 6 \\
\hline $\mathrm{Ba}\left(\mathrm{S}_{0.77}, \mathrm{Cr}_{0.23}\right) \mathrm{O}_{4}$ & 10.1 & Rai et al. (1986) & Type 6 \\
\hline $\mathrm{Cd}(\mathrm{OH})_{2(\mathrm{ss})}$ & -13.7 & Meima and Comans (1998) & Thermo \\
\hline $\mathrm{Pb}(\mathrm{OH})_{2(\mathrm{ss})}$ & -8.2 & $\begin{array}{l}\text { Visual MINTEQ Type } 6 \\
\text { database }\end{array}$ & Thermo \\
\hline $\mathrm{Cu}(\mathrm{OH})_{2(\mathrm{ss})}$ & -10.7 & $\begin{array}{l}\text { Visual MINTEQ Type 6 } \\
\text { database }\end{array}$ & Thermo \\
\hline $\mathrm{Fe}_{2}\left(\mathrm{MoO}_{4}\right)_{3(\mathrm{ss})}$ & 35.3 & Dijkstra et al. (2002) & Thermo \\
\hline $\mathrm{FeAsO}_{4}: 2 \mathrm{H}_{2} \mathrm{O}_{(\mathrm{ss})}$ & 20.2 & $\begin{array}{l}\text { Visual MINTEQ Type 6 } \\
\text { database }\end{array}$ & Thermo \\
\hline
\end{tabular}

Table 4 Pure solid phases used in Modes 3 and 4

Chemical Species $\quad \log \mathrm{K}$

$\begin{array}{ll}\mathrm{Pb} 3(\mathrm{VO} 4) 2 & -6.14\end{array}$

BaHAsO4:H2O 24.64

$\mathrm{SiO} 2(\mathrm{am}, \mathrm{gel}) \quad 2.71$

Dolomite (disordered) $\quad 16.54$

$\begin{array}{ll}\mathrm{Ba}(\mathrm{S} 0.96 \mathrm{Cr} 0.04) 04 & 9.79\end{array}$ 


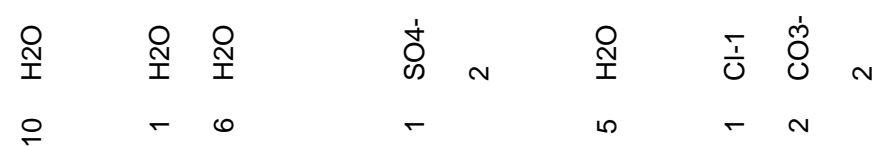

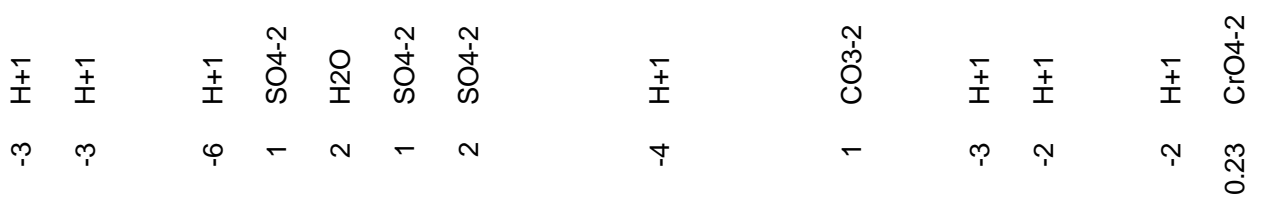

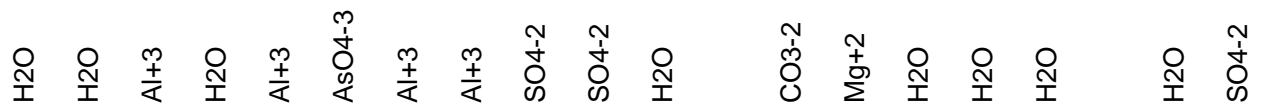

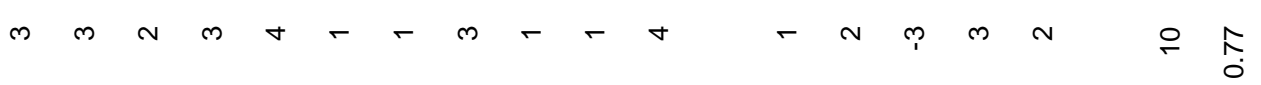

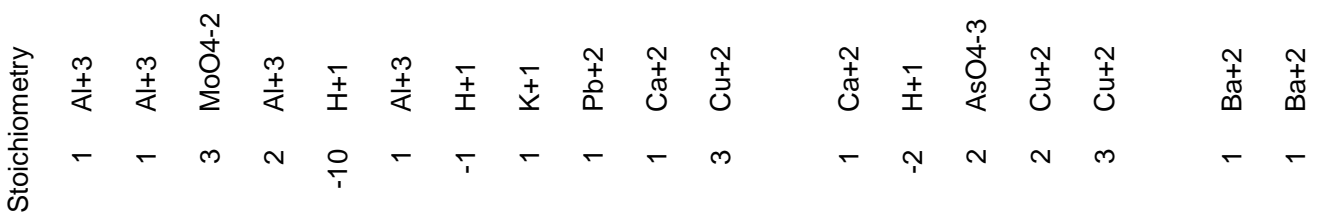

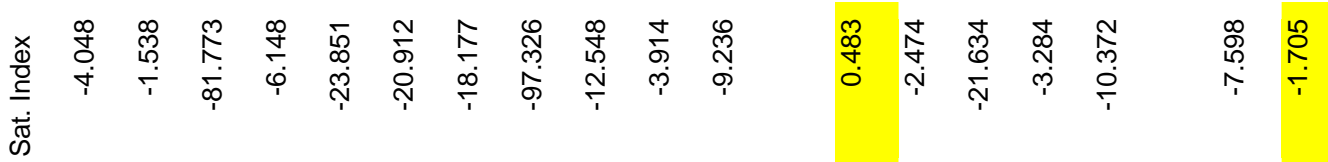

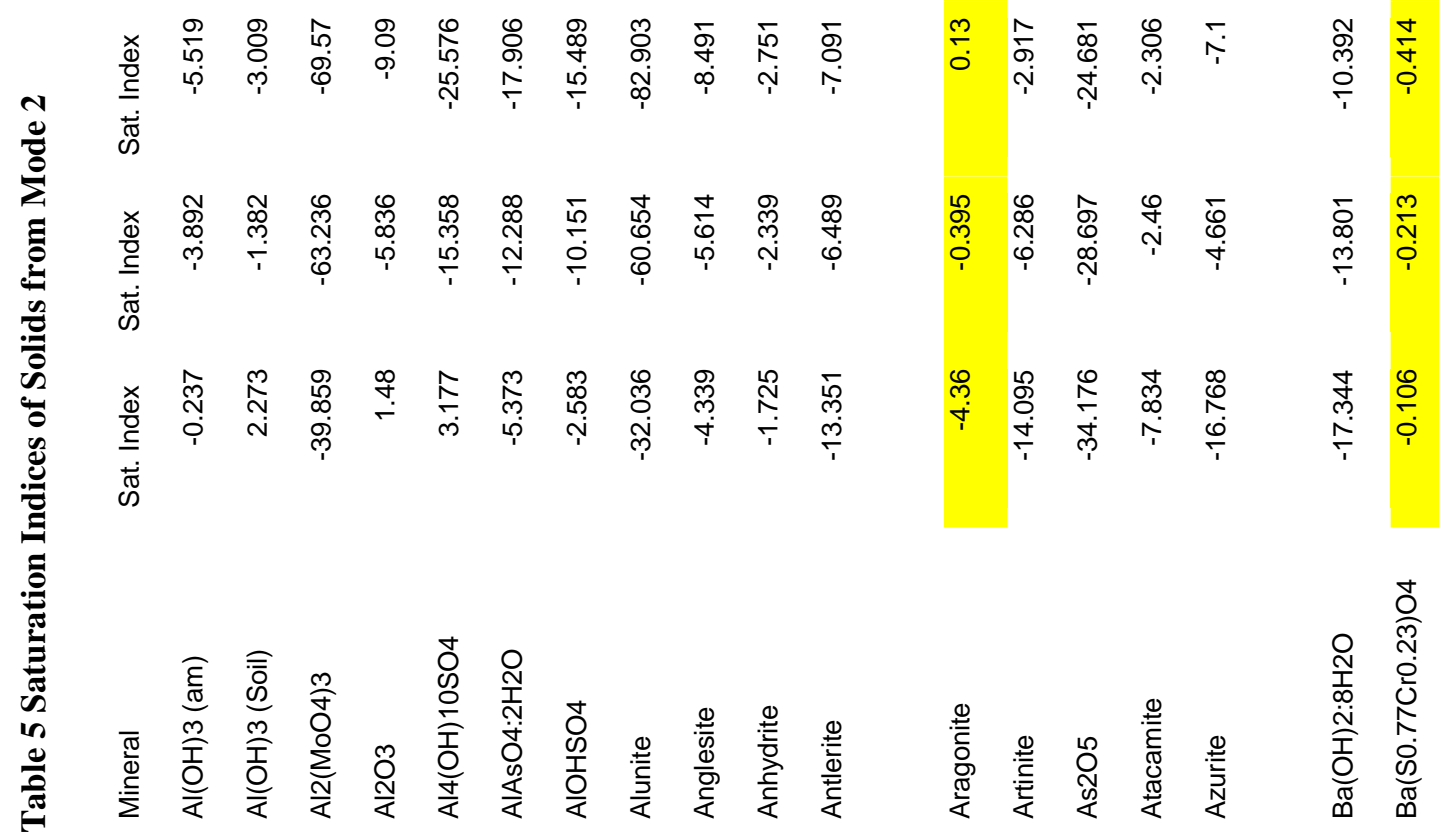




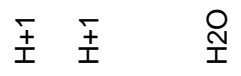

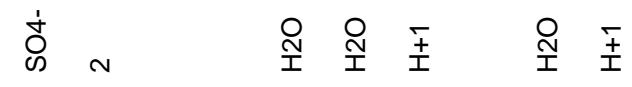

$$
\begin{aligned}
& \varphi+
\end{aligned}
$$

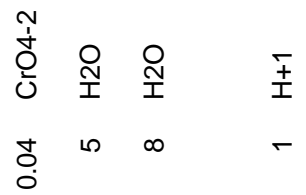

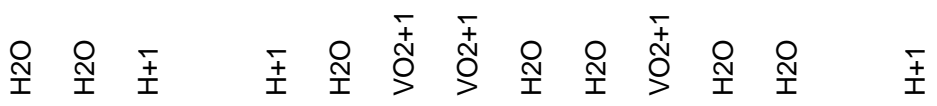

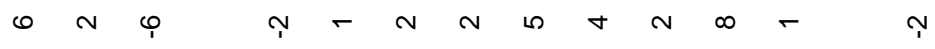

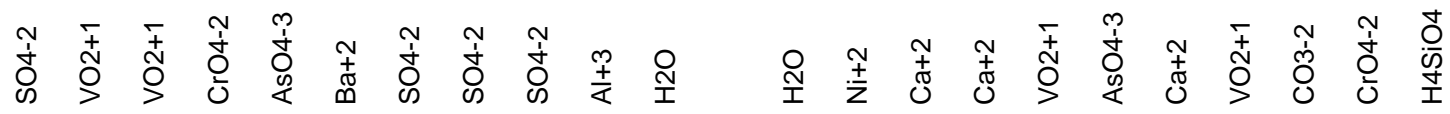

$$
\begin{aligned}
& \text { go }
\end{aligned}
$$

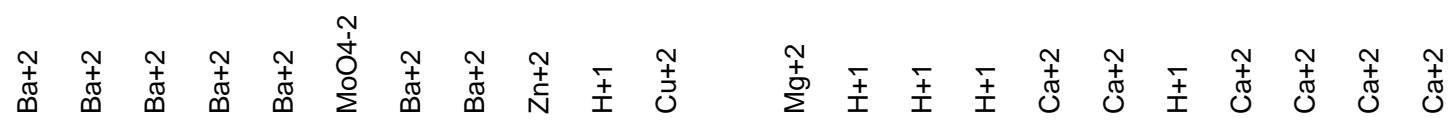

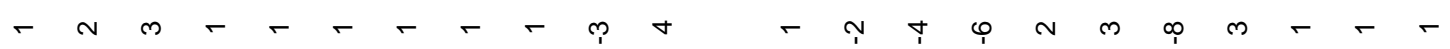

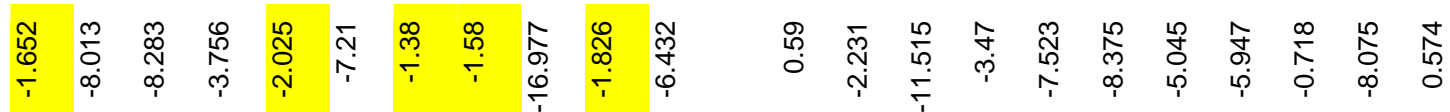

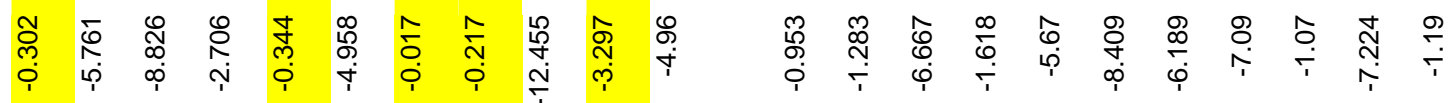

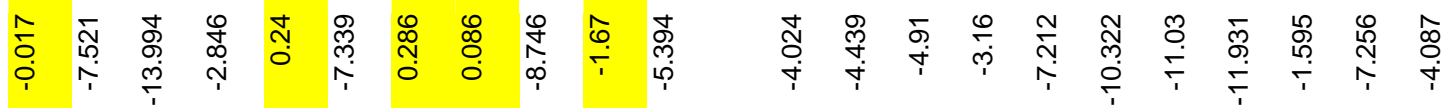

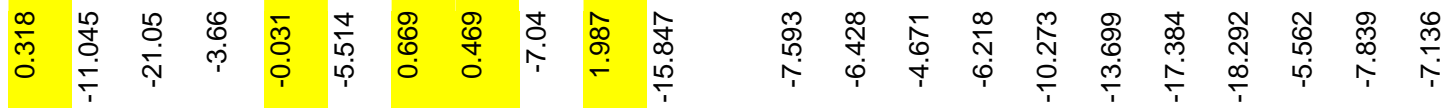

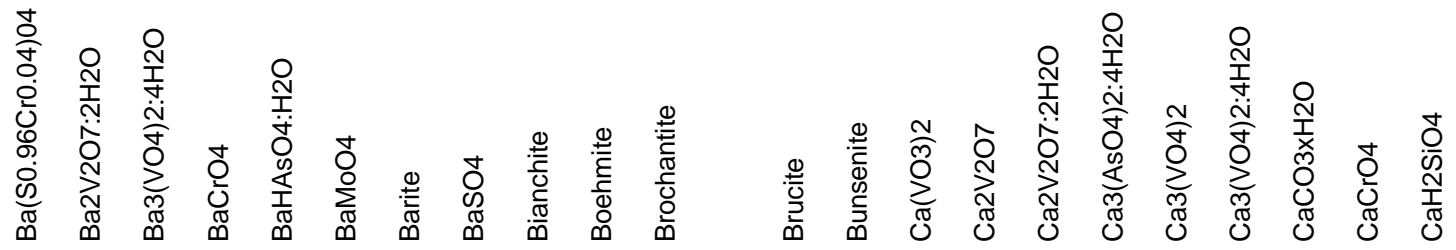




$$
\begin{aligned}
& \text { 䓪 a } \\
& \overline{\overline{0}} \quad \bar{I} \\
& \begin{array}{llll}
- & & - & -
\end{array}
\end{aligned}
$$

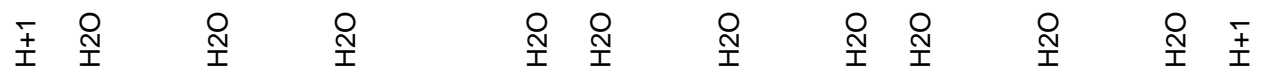

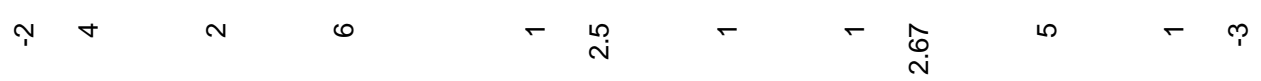

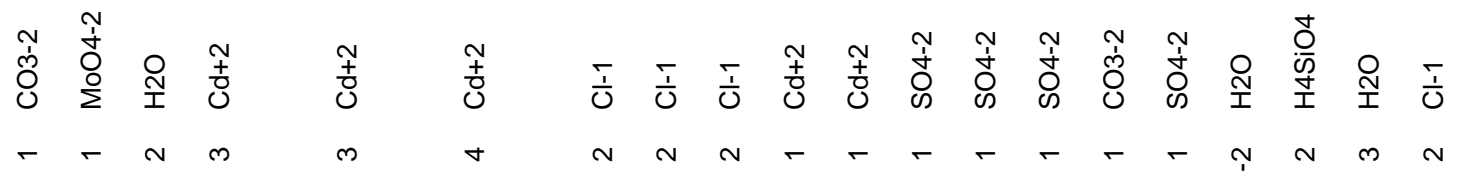

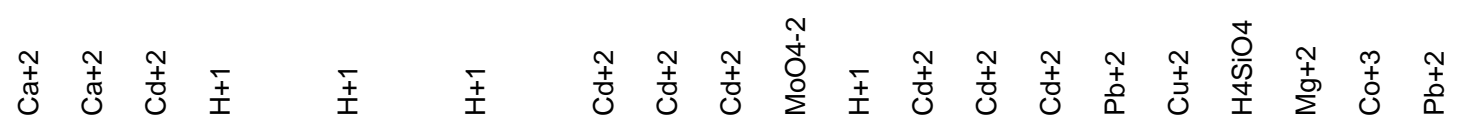

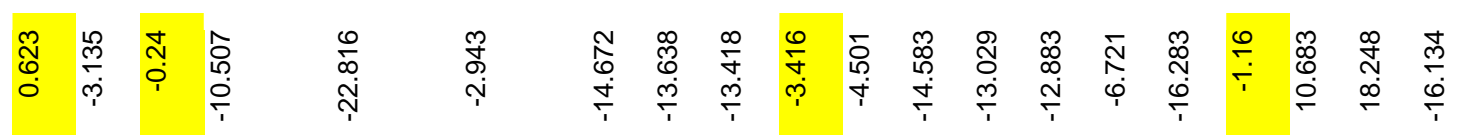

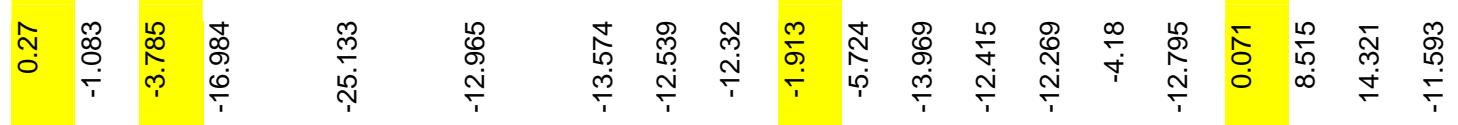

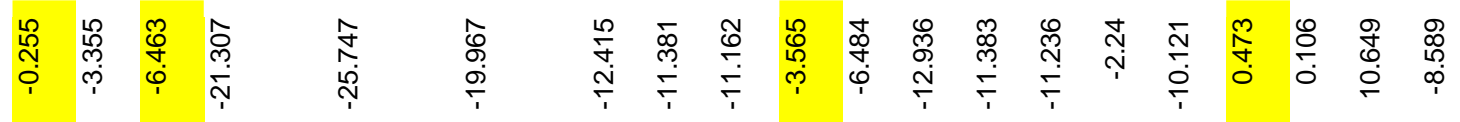

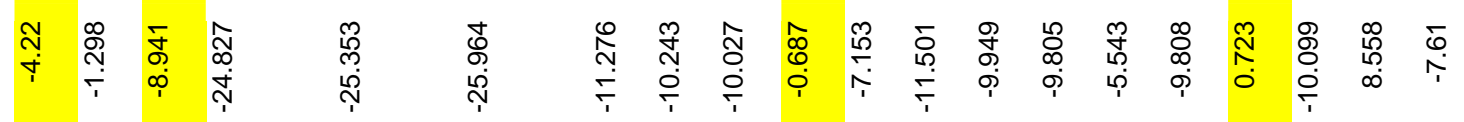

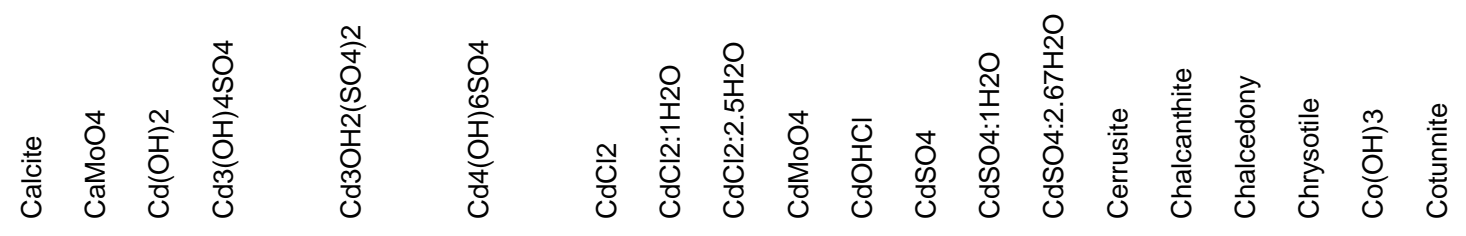




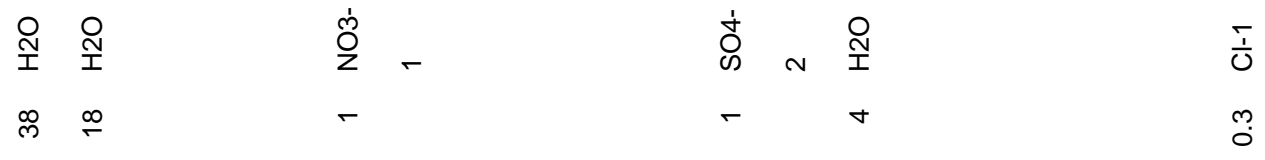

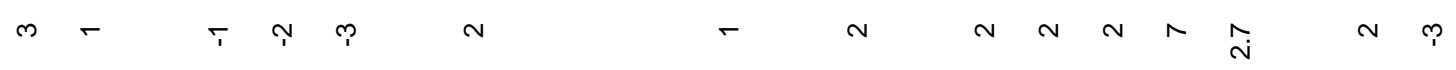

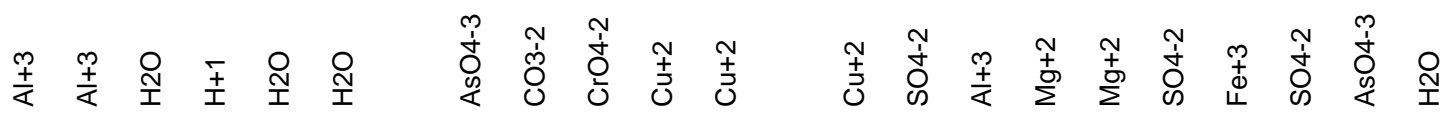

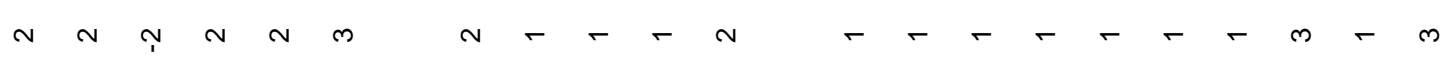

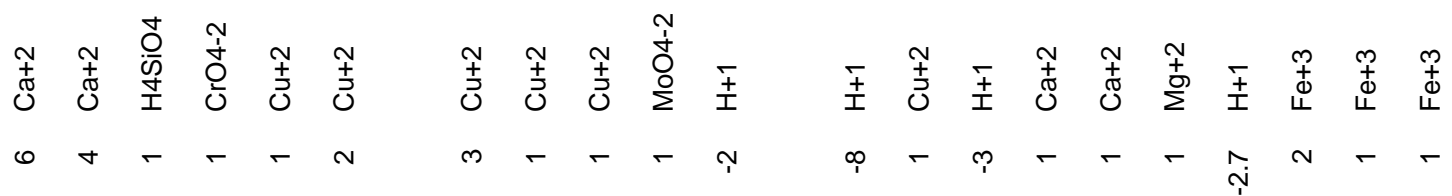

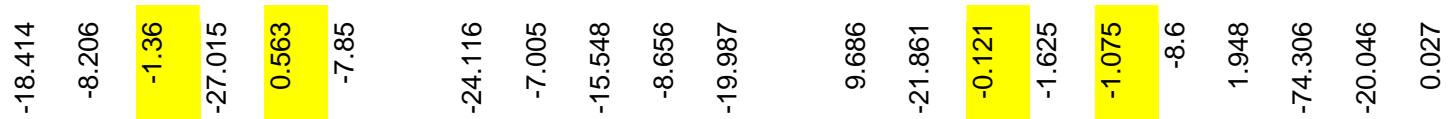

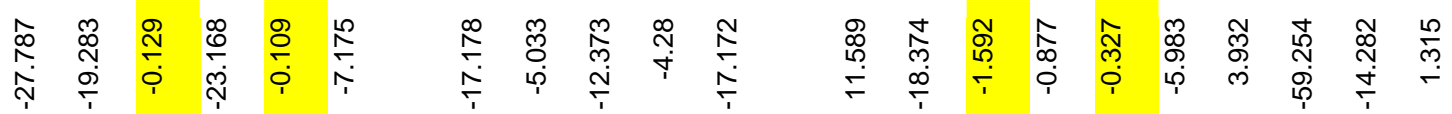

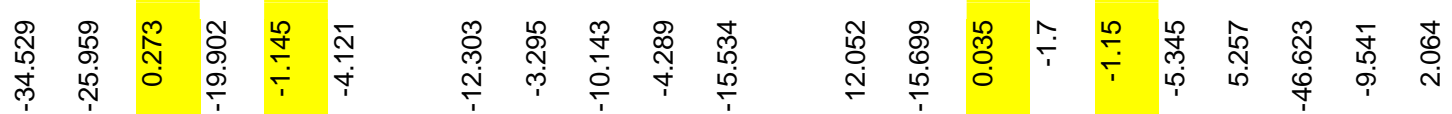

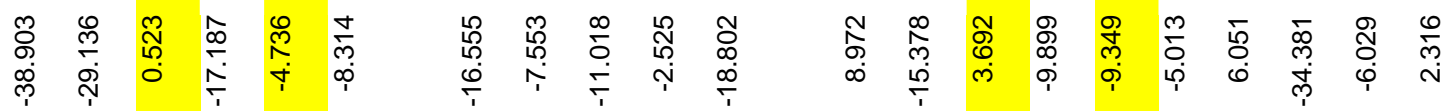

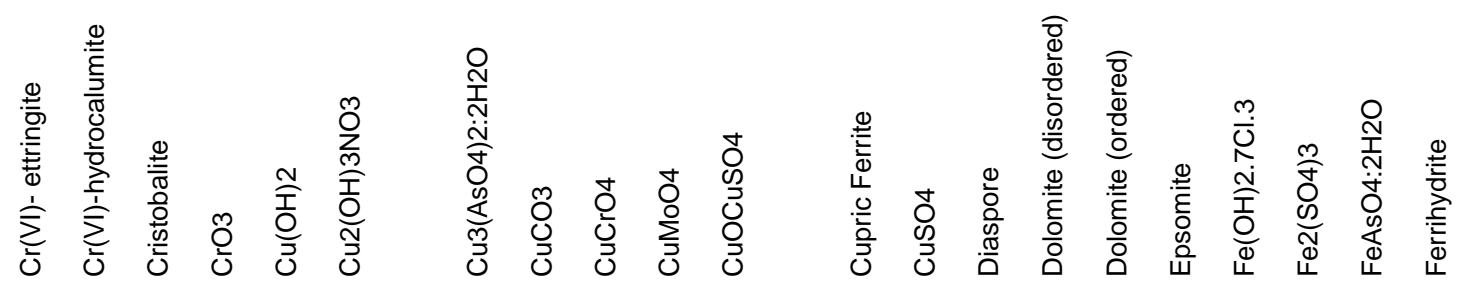




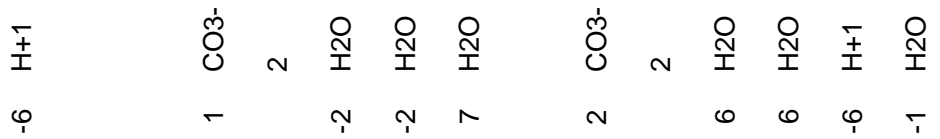

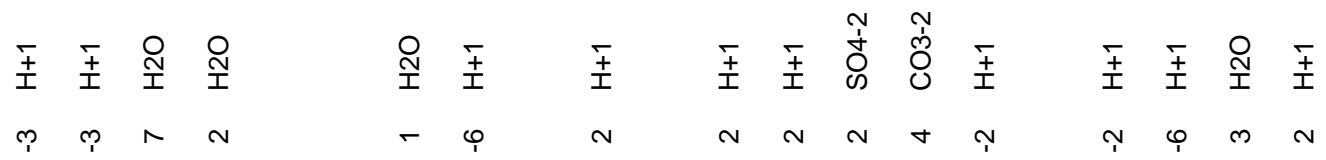

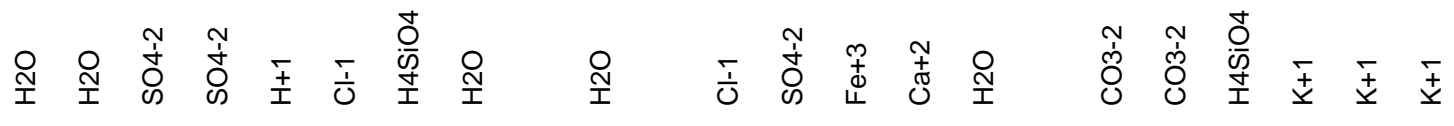

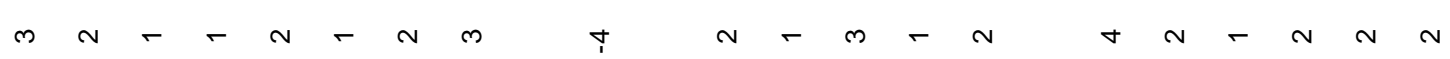

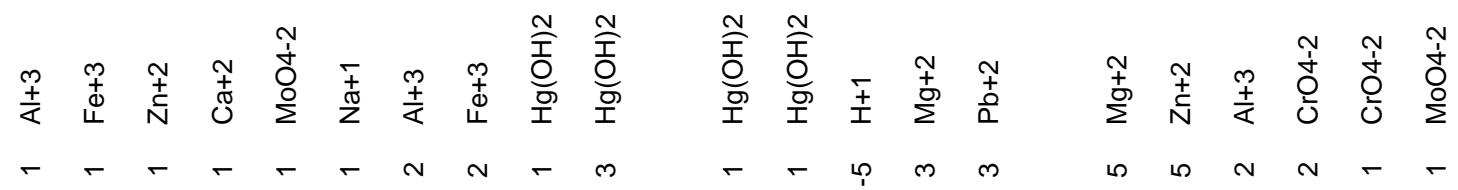

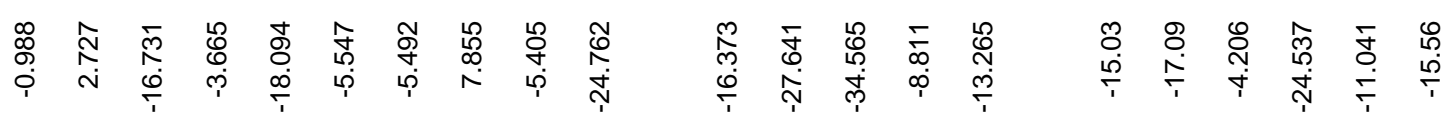

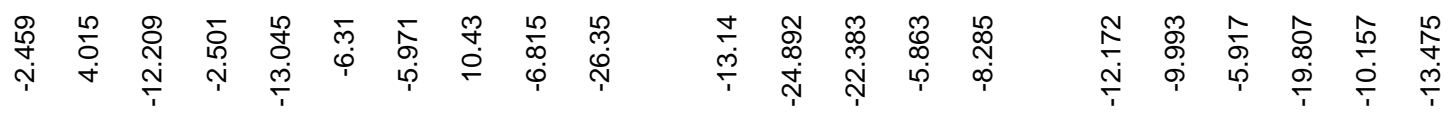

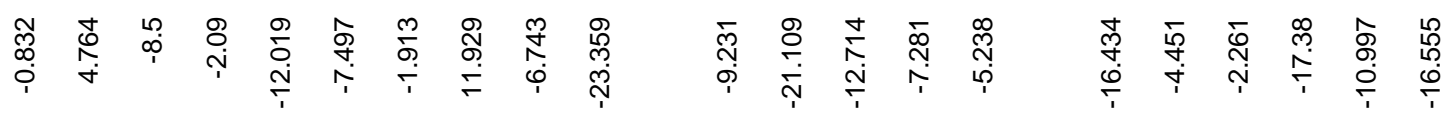

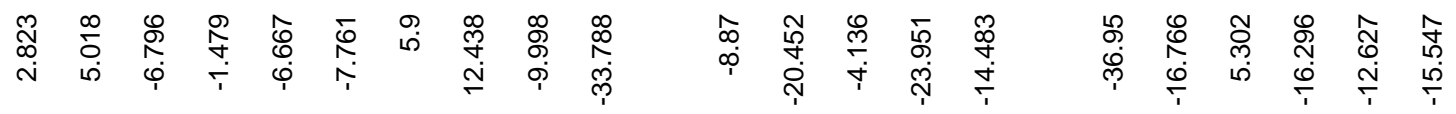

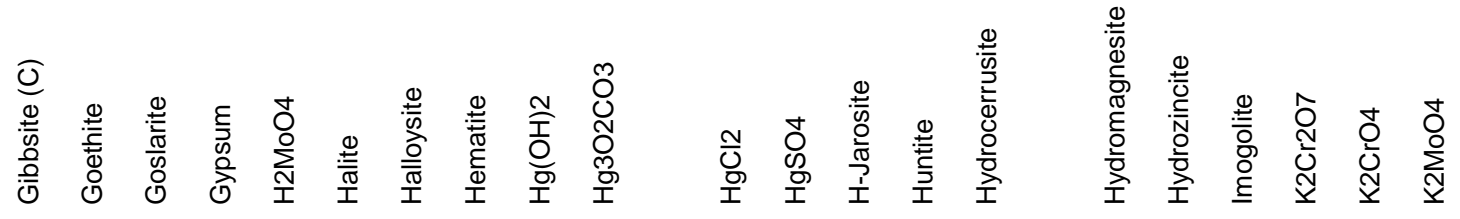




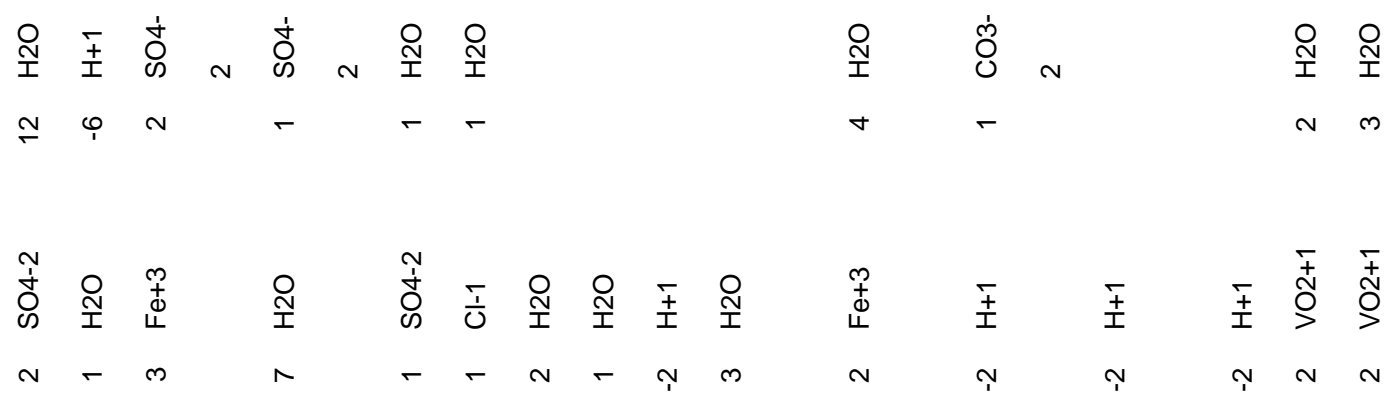

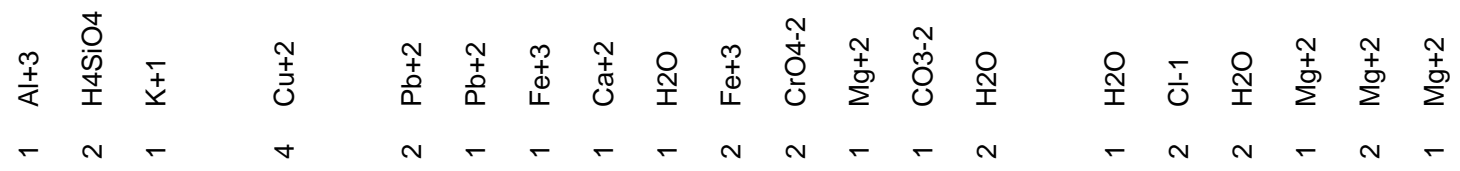

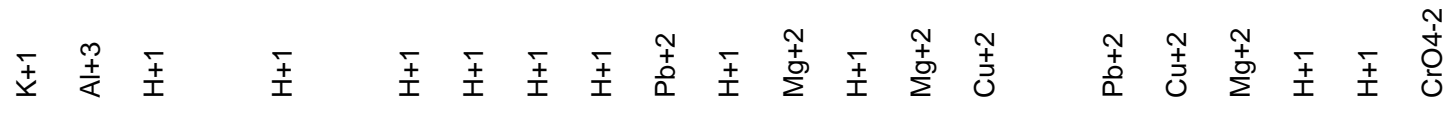

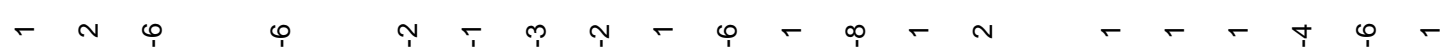

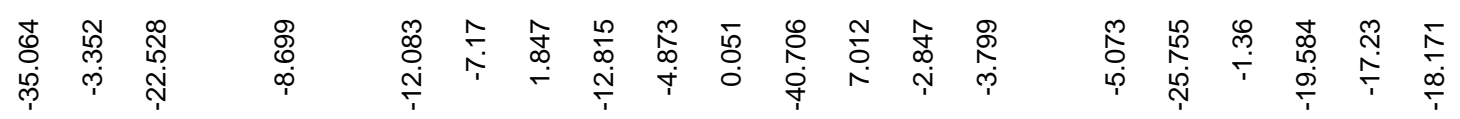

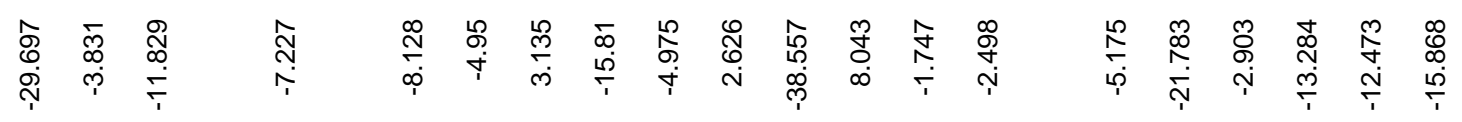

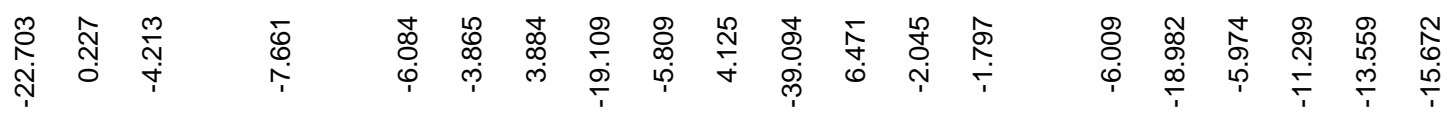

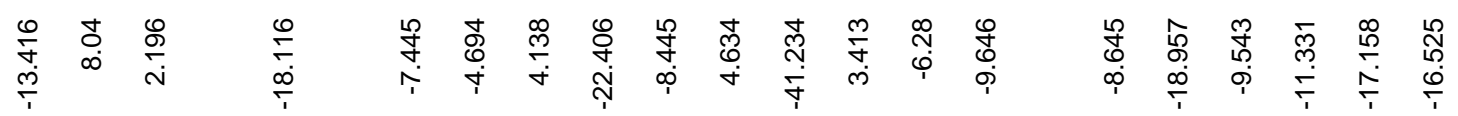

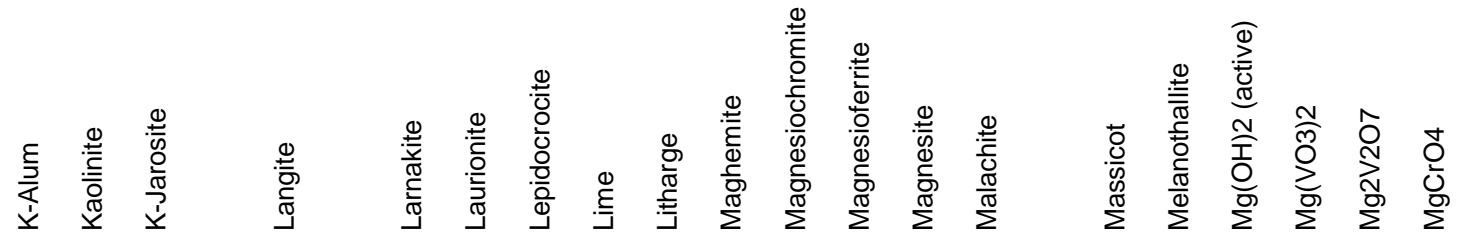




$$
\begin{aligned}
& \text { ำ }
\end{aligned}
$$

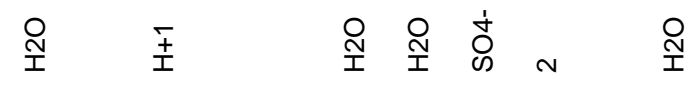

$$
\begin{aligned}
& \sim
\end{aligned}
$$

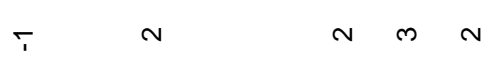

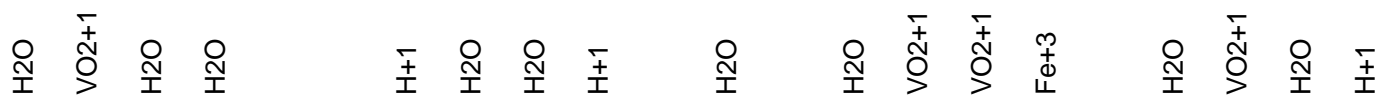

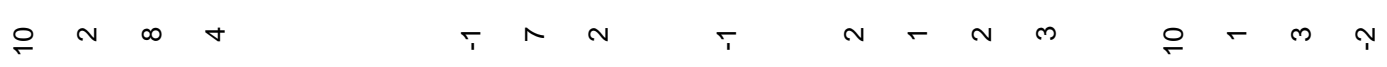

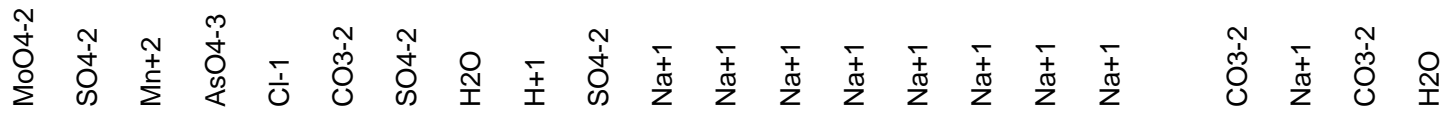

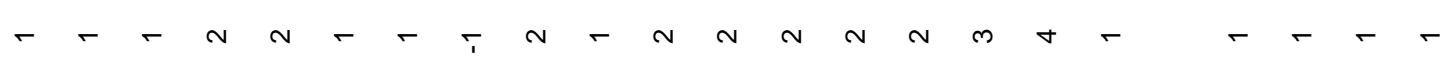

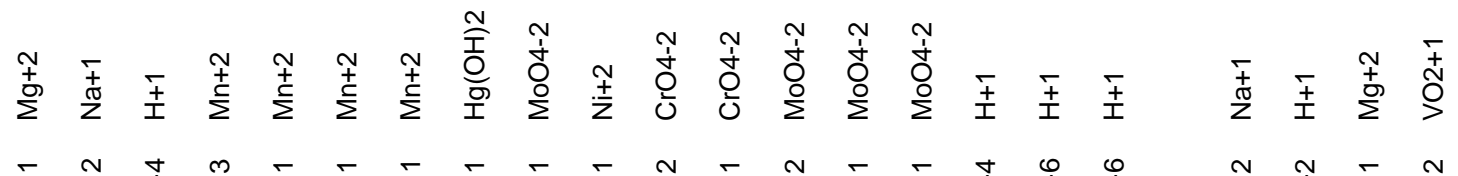

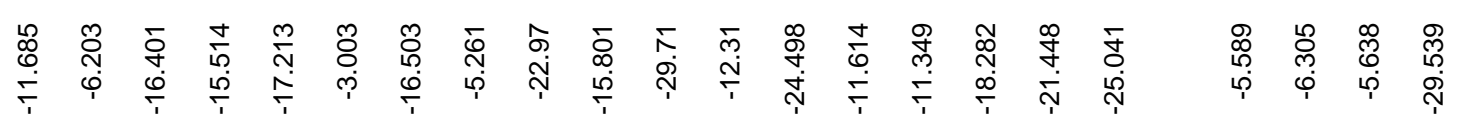

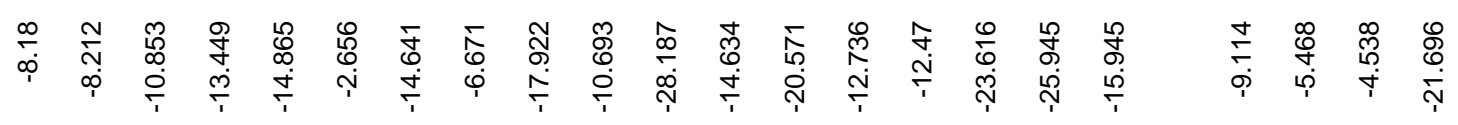

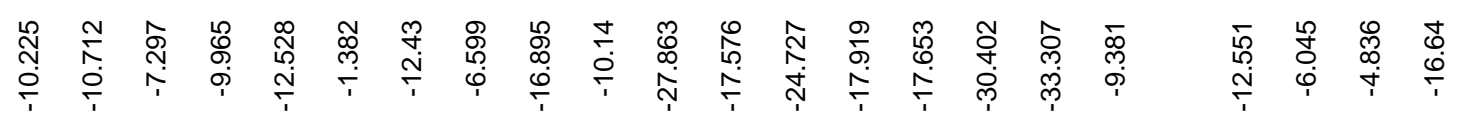

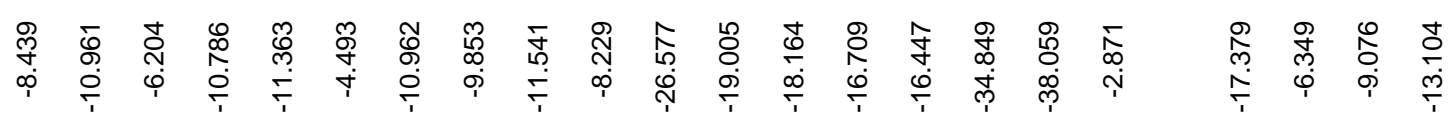

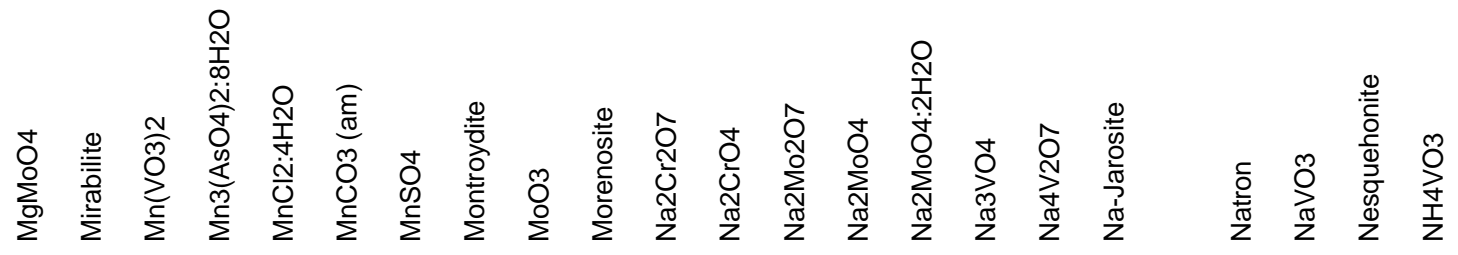




$$
\begin{aligned}
& \text { 空至它 }
\end{aligned}
$$

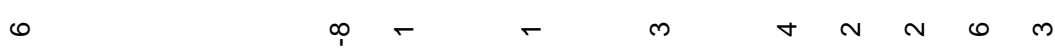

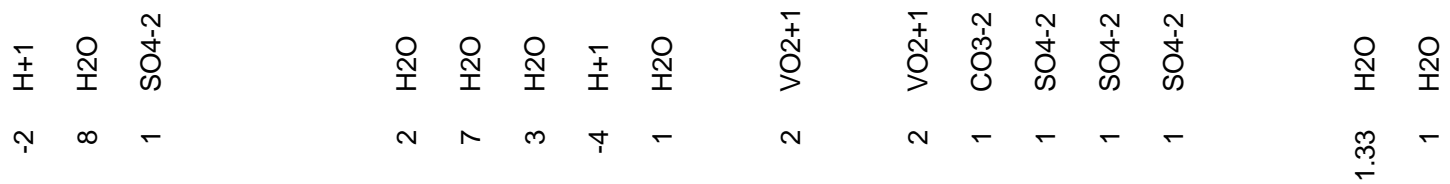

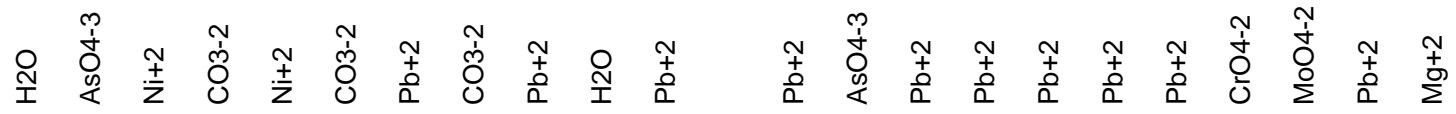

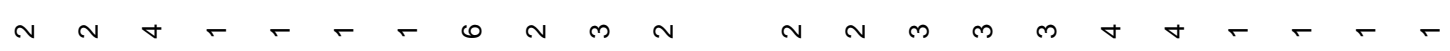

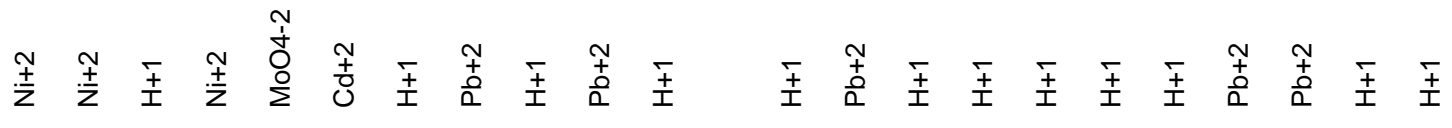

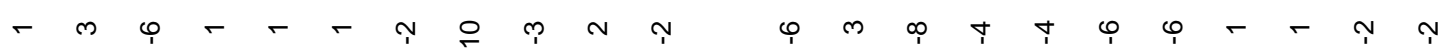

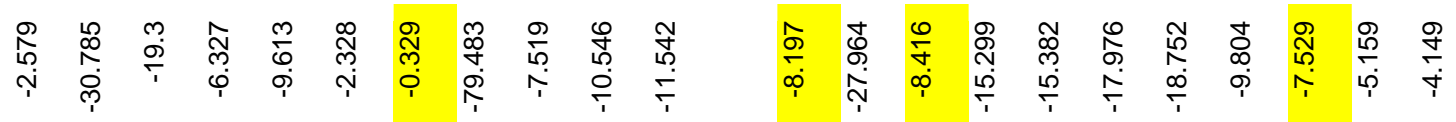

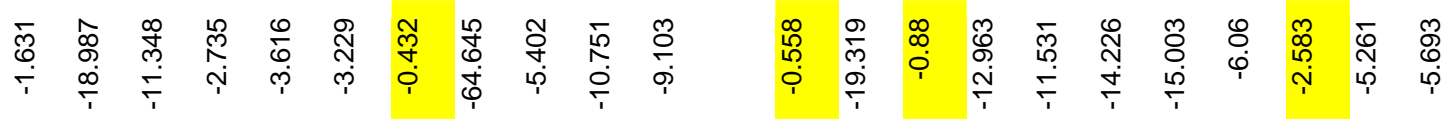

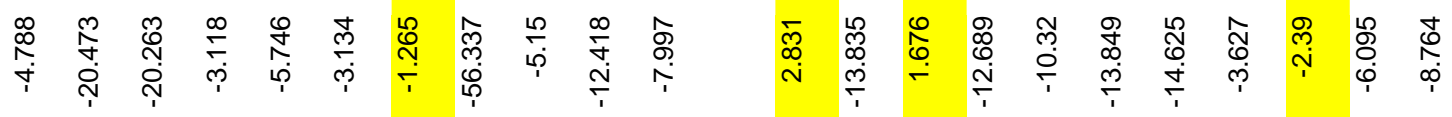

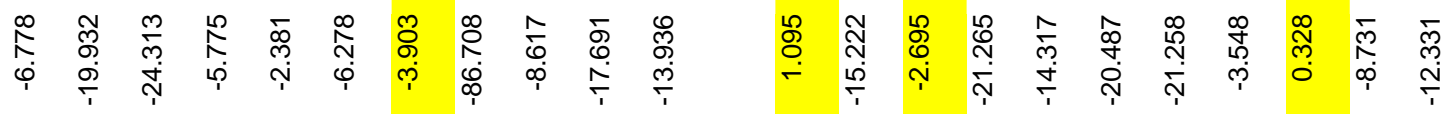

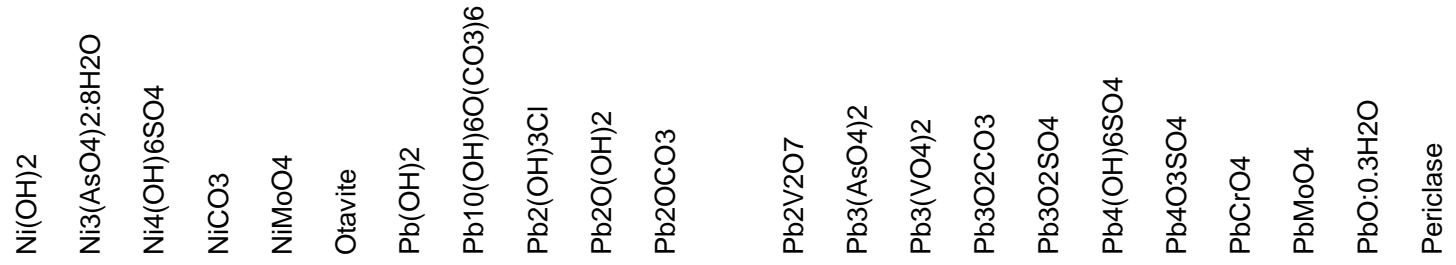




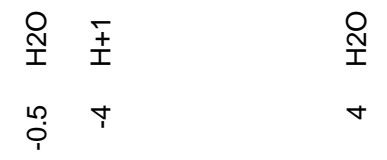

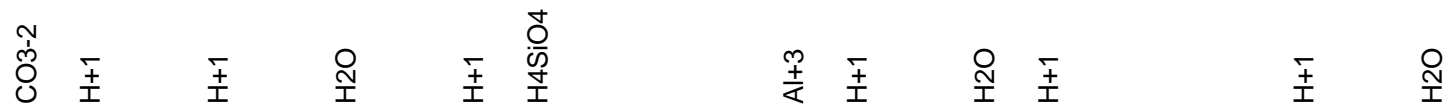

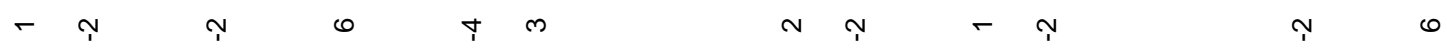

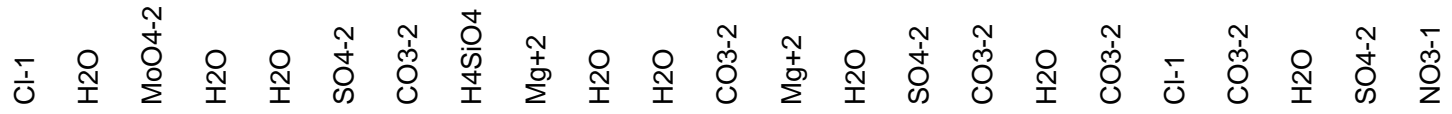

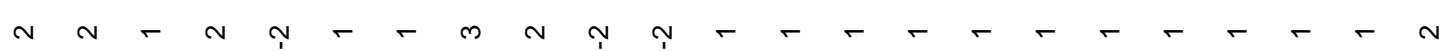

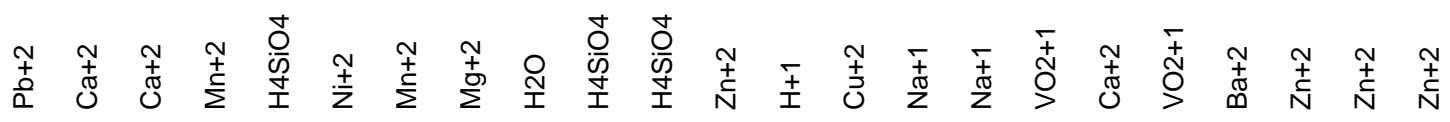

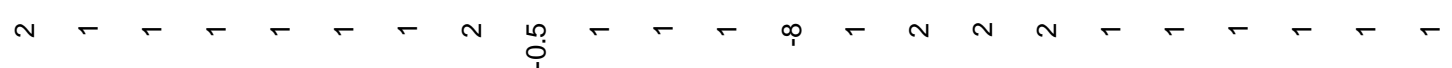

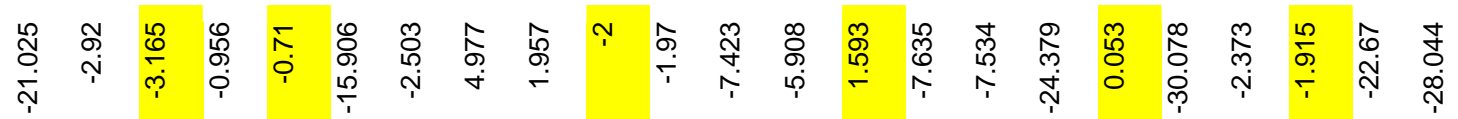

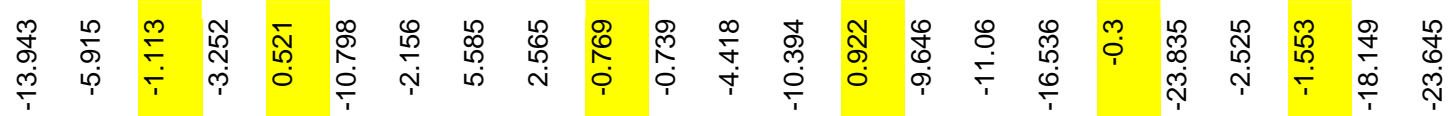

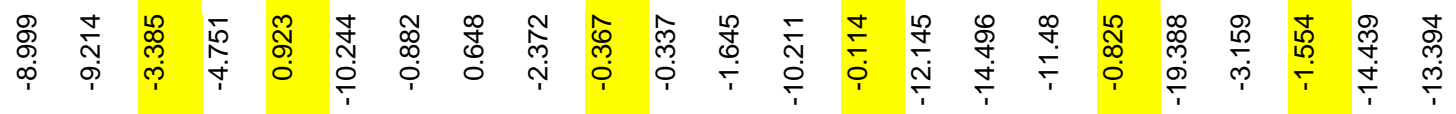

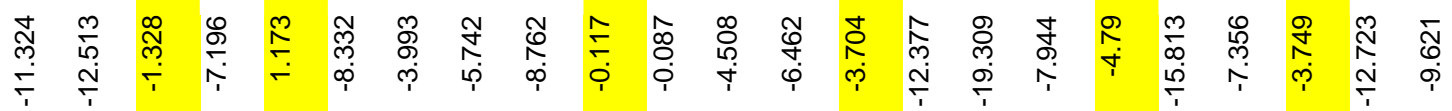

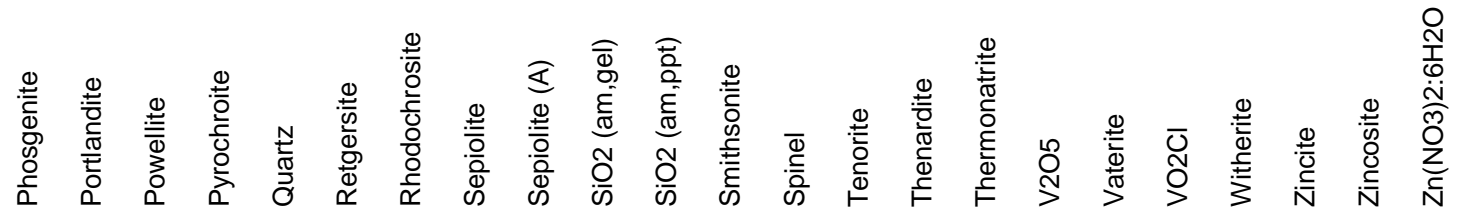




$$
\begin{aligned}
& \text { 雚 a }
\end{aligned}
$$

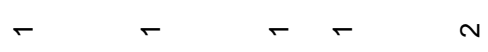

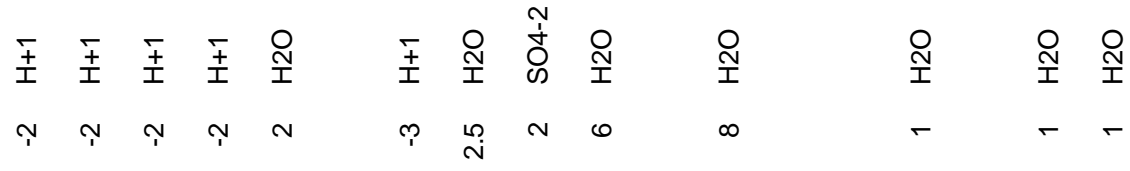

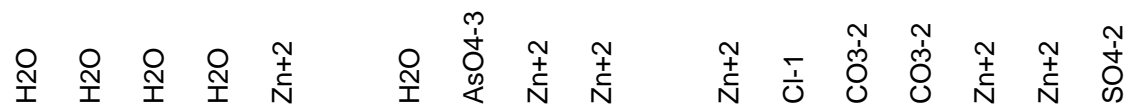

$$
\begin{aligned}
& \sim \sim \sim \sim \sim \sim \sim c
\end{aligned}
$$

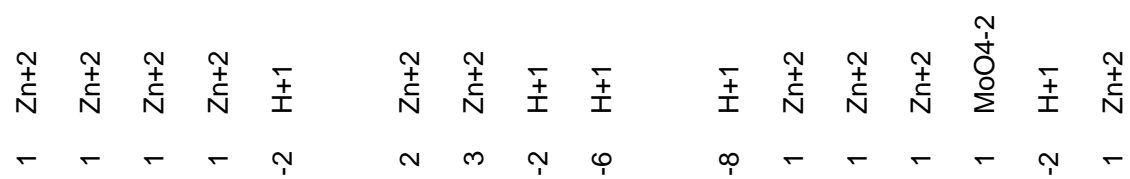

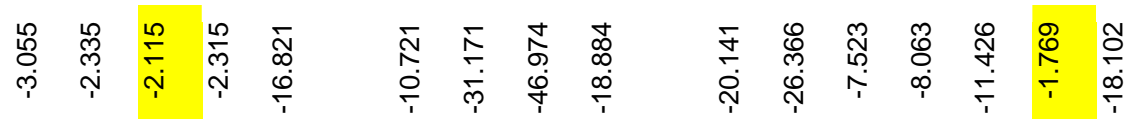

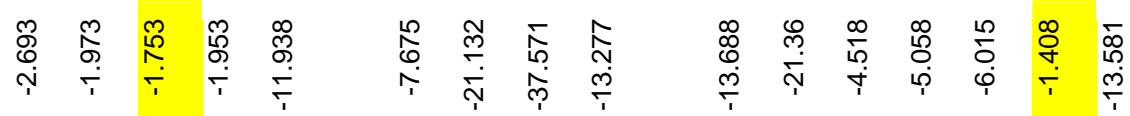

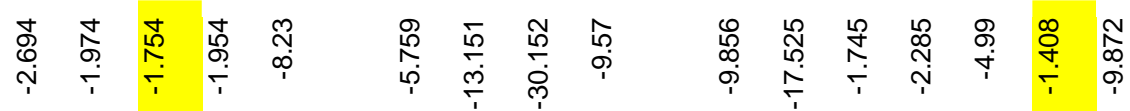

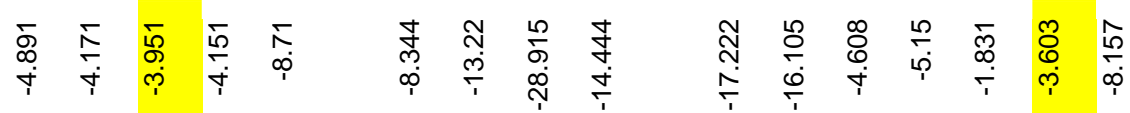

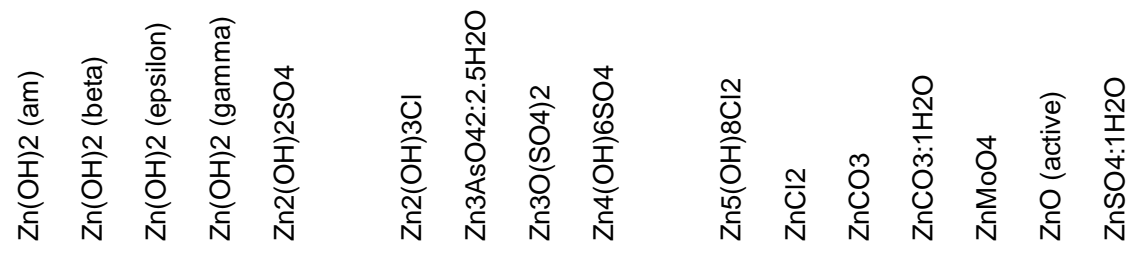




\section{$\underline{\text { References }}$}

Fällman, A.-M., Ph.D. Thesis: Characterization of residues: Release of contaminant from slags and ashes. Department of physics and measurement technology. Linkoping, Linkoping University, 1997.

Fällman, A.-M., Eighmy, T.T., and Salaneck, W.R. (1999) Aging reactions in residues. Stockholm, Swedish Environmental Protection Agency.

Fuller, C.C., J.R. Bargar, J.A. Davis, M.J. Piana, "Mechanisms of Uranium Interactions with Hydroxyapatite: Implications for Groundwater Remediation”, Environmental Science and Technology 36: 158-165 (2002).

NEN 7341, Determination of leaching characteristics of inorganic components from granular waste materials, NNI, Delft (Netherlands), 1993

Lower, S.K., P.A. Maurice, S.J. Traina, E.H. Carlson, “Aqueous Pb Sorption by Hydroxylapatite: Applications of Atomic Force Microscopy to Dissolution, Nucleation, and Growth Studies”, American Mineralogist 83: 147-158 (1998).

Rai, D., Zachara, J.M., Schwab, A.P., Schmidt, R.L., Girvin, D.C., and Rogers, J.E. Chemical attenuation rates, coefficients and constants in leachate migration. Volume 1: A critical review. EPRI EA-3356, Electrical Power Research Institute. Palo Alto, 1986 
Stipp, S.L., M.F. Hochella Jr., G.A. Parks, J.O. Leckie, " $\mathrm{Cd}^{2+}$ Uptake by Calcite, Solidstate Diffusion, and the Formation of Solid-solution: Interface Processes Observed with Near-surface Sensitive Techniques (XPS, LEED, and AES)", Geochimica et Cosmochimica 56: 1941-1954 (1992).

Sposito, G, "Distinguishing Adsorption from Surface Precipitation", in Geochemical Processes at Mineral Surfaces, American Chemical Society Symposium Series: 217-228 (1986).

Zachara, J.M., J.A. Kittrick, L.S. Dake, J.B. Harsh, , “Solubility and Surface Spectroscopy of Zinc Precipitates on Calcite", Geochimica et Cosmochimica 53: 9-19 (1989). 